\section{INFLUENCE OF THE POROUS MEDIA ON HEAT EXCHANGE AT FILM BOILING LIQUID}

\section{Avramenko A.A., Kovetskaya M.M., Dmitrenko N.P., Kovetska, Yu.Yu.}

Institute of Engineering Thermophysics, National Academy of Sciences of Ukraine, 2a, Kapnist str.,Kiev 03680,

Ukraine

$$
\text { https://doi.org/10.31472/ttpe.3.2021.1 }
$$

The present work focuses on a study of heat transfer during film boiling of a liquid on a vertical heated wall immersed in a porous medium subject to variation of different parameters of the porous medium and heating conditions at the wall. An analytical solution was obtained for the problem using Darcy-Brinkman-Forchheimer model. It was shown that heat transfer intensity during film boiling in a porous medium is weaker than in a free fluid (without porosity) and decreases with the decreasing permeability of the porous medium.

The use of a porous medium model in the DarcyBrinkman-Forchheimer approximation showed the effect of the Forchheimer parameter on heat transfer during film boiling in a porous medium. An increase in the Forchheimer parameter leads to heat transfer deterioration, which is more significant at small values of the Darcy number. Effects of different thermal boundary conditions on the heated wall on the heat transfer are insignificant.

References 18, tables 2, figures 1.

Key words: heat transfer, film boiling of liquid, porous medium.

1. Singh H., Myong R.S. Critical Review of Fluid Flow Physics at Micro- to Nano-scale Porous Media Application in the Energy Sector. Advances in Materials Science and Engineering. 2018. Vol. 1. P.1-31

2. Alomar O.R., Trimis D., Mendes M., Ray S. Numerical simulation of complete liquid-vapour phase change process inside porous media: A comparison between local thermal equilibrium and non-equilibrium models", Int. J. of Thermal Sciences. 112 (2017) $222-241$.

3. Wang C.Y. A fixed-grid numerical algorithm for twophase flow and heat transfer in porous media. Numerical Heat Transfer.1997. 32:1. P.85- 105

4. Alomar O.R., Mendes M.A.A., Trimis D., Ray S. Numerical simulation of complete liquidevapour phase change process inside porous media using smoothing of diffusion coefficient. Int. Journal of Thermal Sciences.2014. 86. P. $408-420$

5. Alomar O.R., Mendes M.A.A., Trimis D., Ray S. Simulation of complete liquid-vapor phase change inside divergent porous evaporator. International Journal of Materials, Mechanics and Manufacturing. 2014. Vol. 2, No. 3
6. Duval F., Fichot F., Quintard M. A local thermal nonequilibrium model for two-phase

flows with phase-change in porous media //International Journal of Heat and Mass Transfer. 2004. 47. P. 613-639

7. Bachrata A. , Fichot F. , Quintard M., Repetto G. , Fleurot J. Non-Local Equilibrium Two-phase Flow model With Phase Change In Porous Media And Its Application To Refolding Of a Severely Damaged Reactor Core. Porous Media and Its Applications in Science, Engineering and industry. AIP Conf. Proc. 2012. 1453. P.147- 152

8. Alomar O.R., Mohammed R.R., Mendes M.A.A., Ray $S$., Trimis $D$. Numerical investigation of two-phase flow in anisotropic porous evaporator. Int. J. of Thermal Sciences. 2019.135. P. 1 - 16 .

9. Alazmi B., Vafai K. Analysis of Variable Porosity, Thermal Dispersion, and Local Thermal Non-Equilibrium on Free Surface Flows Through Porous Media. Journal of Heat Transfer. 2004. V.126. P.389-399

10. Avramenko, A.A., Tyrinov, A.I., Shevchuk, I.V., Dmitrenko N.P., Kravchuk A.V. Mixed convection in a vertical flat microchannel. International Journal of Heat and Mass Transfer. 2017. Vol. 106. P. 1164-1173

11. Avramenko, A.A., Shevchuk, I.V., Moskalenko, A.A., Lohvynenko, P.N., Kovetska, Yu.Yu. Instability of a vapor layer on a vertical surface at presence of nanoparticles. Applied Thermal Engineering. 2018. Vol. 139. P. 87-98.

12. Avramenko, A.A., Tyrinov, A.I., Shevchuk, I.V., Dmitrenko N.P. Dean instability of nanofluids with radial temperature and concentration non-uniformity. Physics of Fluids. 2016. Vol. 28 (3). P.034104

13. Avramenko A.A., Kovetskaya M.M., Kondratieva E.A., Sorokina T.V. [Heat transfer for filn boiling of a liquid on a vertical heated wall in a porous medium]. Thermophysics and Thermal Power Engineering. 2021. V. 43, №1. P. 20-29. (in Ukr.)

14. Nield, D.A., Bejan, A. Convection in Porous Media. 3th Edition. New York: Springer. 2006.

15. Bromley, L.A. Heat transfer in stable film boiling. Chem. Eng. Prog. 1950. Vol. 46. P. 211-227.

16. Olver, P.J. Applications of Lie groups to differential equations. Springer- Verlag: New York Berlin Hieidelberg. 1993.

17. Kamke, E. Differentialgleichungen: Lösungsmethoden und Lösungen, I, Gewöhnliche Differentialgleichungen, B. G. Teubner, Leipzig. 1977.

18. Ellion, M.E. A Study of the mechanism of Boiling heat transfer. Jet Prop. Lab. Memo, CIT. 1954. 20, P. 1- 88. 
УДК: 536.242

\section{ОСОБЛИВОСТІ ПРОСТОРОВОГО РОЗПОДІЛУ ПИТОМОЇ ТЕПЛОєМНОСТІ НАДКРИТИЧНОЇ ВОДИ ПРИ ІІЇ ТЕЧІЇ У ВЕРТИКАЛЬНИХ ГЛАДКИХ ТРУБАХ}

Фіалко Н.М. ${ }^{1}$, член-кореспондент НАН України, Носовський А.В. ${ }^{2}$, академік НАН України, Альошко С.О.1, канд. техн. наук, Піоро І.Л.․ㅜ, доктор технічних наук, Хміль Д.П. ${ }^{1}$

${ }^{1}$ Інститут технічної теплофізики НАН України, вул. Марії Капніст, 2а, м. Київ, 03057, Украӥна

${ }^{2}$ Інститут проблем безпеки АЕС НАН України, Київ, Україна, вул. Лисогірська, 12, м. Київ 02000, Украӥна

${ }^{3}$ Faculty of Energy Systems and Nuclear Science University of Ontario Institute of Technology 2000 Simcoe Str. N., Oshawa ON L1K 7 K4 Canada

https://doi.org/10.31472/ttpe.3.2021.2

Наводяться результати комп'ютерного моделювання просторового розподілу питомої теплоємності надкритичної води при іiі висхідній течії у вертикальних гладких трубах. Представлено дані порівняльного аналізу цього розподілу при різних значеннях питомого теплового потоку, що підводиться до стінки труби.
Приводятся результаты компьютерного моделирования пространственного распределения удельной теплоемкости сверхкритической воды при ее восходящем течении в вертикальных гладких трубах. Представлены данные сравнительного анализа этого распределения при различных значениях удельного теплового потока, подводимого к стенке трубы.
The results of computer modeling of the spatial distribution of the specific heat capacity of supercritical water during its ascending flow in vertical bare tubes are given. The data of a comparative analysis of this distribution at various values of the specific heat flux supplied to the tubes wall are presented.

Бібл. 15 , рис. 4.

Ключові слова: CFD моделювання, теплоємність надкритичної води, змішана конвекція.

$c_{p}$ - питома теплоємність, кДж/(кг॰К);

$d$ - внутрішній діаметр, м;

$G$ - масова швидкість, кг/(м² $\mathrm{c})$;

$L$ - ненагрівана довжина труби, м;

$L_{1}$ - нагрівана довжина труби, м;

$P$ - тиск, МПа;

$q$ - густина теплового потоку на стінці

труби, кВт/м²;

$r$ - радіальна координата;

$T$ - температура, ${ }^{\circ} \mathrm{C}$;

$T u$ - інтенсивність турбулентності, \%;

$x$ - осьова координата, м;

\section{Bcmyn}

У теплоенергетиці розвинутих країн світу, як відомо, переважають енергоблоки надкритичного тиску. Це зумовлено, насамперед, їх високою тепловою ефективністю. Перевищення термічного коефіцієнта корисної дії цих блоків у порівнянні з відповідними блоками на докритичних параметрах може сягати більш ніж $10 \%$. Один 3 перспективних напрямів розвитку атомної енергетики також пов'язаний 3 переходом на надкритичні параметри.

Здійснення такого переходу потребує вирішення низки задач реакторної теплофізики. Зокрема, важливими $є$

\section{Нижні індекси:}

вx- вхідний;

pc - псевдокритичний перехід «псевдорідина-

псевдогаз» (pseudocritical);

\section{Скорочення:}

CFD - (Computational Fluid Dynamics) розрахункова гідродинаміка;

SST - (Shear Stress Transport turbulent model) модель турбулентності переносу зсувних напруг.

дослідження тепломасопереносу при течії надкритичної води у нагріваних каналах [1-14]. При цьому значний інтерес становить вивчення картини просторового розподілу теплофізичних властивостей надкритичної води за вказаних умов. Останні, як відомо, значною мірою визначають особливості течії і теплообміну накритичної води 3 огляду на суттєву температурну залежність цих властивостей при надкритичних тисках.

\section{Мета роботи і постановка завдань досліджень}

Мета роботи полягає у вивченні на основі CFD моделювання закономірностей просторового розподіу питомої теплоємності надкритичної води за умов 
iii висхідної течії в нагріваних вертикальних гладких трубах. При цьому в задачу дослідження входило зіставлення особливостей вказаного розподілу при різних значеннях густини теплового потоку, що підводиться до стінки труби.

В ході досліджень розв'язанню підлягала вісесиметрична задача змішаної конвекції, що відповідає наявності вимушеного і вільного руху надкритичної води. Для забезпечення гідродинамічної стабілізаціїтечії на вході в трубу перед ним встановлювалась ненагрівана ділянка довжиною $L_{l}$. У вхідному перерізі труби задавались постійні значення масової швидкості $G$, температури $T_{6 x}$, тиску $P_{6 x}$ та інтенсивності турбулентності $T u_{6 x}$. У вихідному перерізі ставились «м'які граничні умови». На обтічних водою поверхнях труби задавалися умови прилипання. На ділянці труби, що не обігрівалася, як теплові приймалися умови адіабатичності, на ділянці, що обігрівалася, - умови постійного по довжині труби теплопідводу до іiі стінки.

\section{Методика проведення досліджень.}

Дослідження проводились на основі CFD моделювання з використанням FLUENT коду. Визначення фізичних властивостей надкритичної води здійснювалось за допомогою програми NIST REFPROP, що інтегрувалася у вказаний код [15]. Розрахунки виконувались з подвійною точністю. За результатами верифікації моделей турбулентного переносу використовувалась $k$ - $\omega$ SST модель турбулентності.

При числовій реалізації розв'язку поставленої задачі розрахункова область покривалась нерегулярною сіткою та містила 62400 комірок. Мінімальний крок біля стінки дорівнював $1,5 \cdot 10^{-6}$ м, що відповідало величині $\mathrm{y}^{+}<0,7$.
Розрахункові дослідження виконувались при таких вихідних параметрах: Внутрішній діаметр труби $d=$ $0,01 \mathrm{~m}$; довжина ненагріваної $L$ і нагріваної $L_{1}$ ділянки труби 1,2 м та 4,0 м відповідно; $T_{6 x}=323{ }^{\circ} \mathrm{C} ; P_{\theta x}=24,0 \mathrm{MПа;}$

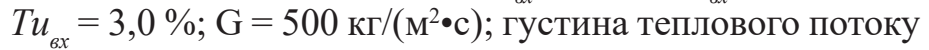

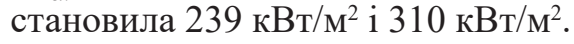

\section{Результати досліджень та їх аналіз}

Характер просторового розподілу питомої теплоємності надкритичної води ср на нагріваній ділянці каналу визначається розподілом температур у даному каналі і температурною залежністю теплоємності ср. (Для досліджених умов остання наведена на рис. 1).

Максимальне значення питомої теплоємності надкритичної води має місце при температурі псевдокритичного переходу Трс, яка для ситуації, що розглядається, становить $381,4{ }^{\circ} \mathrm{C}$. Відтак на просторових розподілах ср положення екстремумів мають відповідати розташуванню ізотерми псевдокритичного переходу $T_{p c}$.

На рисунках 2, 3 наводяться результати CFD моделювання щодо розподілів теплоємності надкритичної води ср по радіусу каналу та його довжині. Тут рис. 2a i 3a відповідають густині теплового потоку $\mathrm{q}=310 \mathrm{\kappa BT} / \mathrm{M}^{2}$, a рис. 26 і $3 б-239$ кВт/м². Рис. 4 ілюструє положення ізотерми псевдокритичного переходу для двох вказаних значень теплового потоку $q$.

Згідно $з$ даними наведеними на рис. 2a, радіальний розподіл $c_{p}$ суттєво змінюється по довжині труби. Так, для $x<x_{p c}$ вказаний розподіл має екстремальний характер (тут $x_{p c}$ - поздовжна координата, яка відповідає перетину ізотермою $T_{p c}$ осі труби). При $x \geq x_{p c}$ максимум кривої $c_{p}=f(r)$ зміщується на вісь труби. На даній

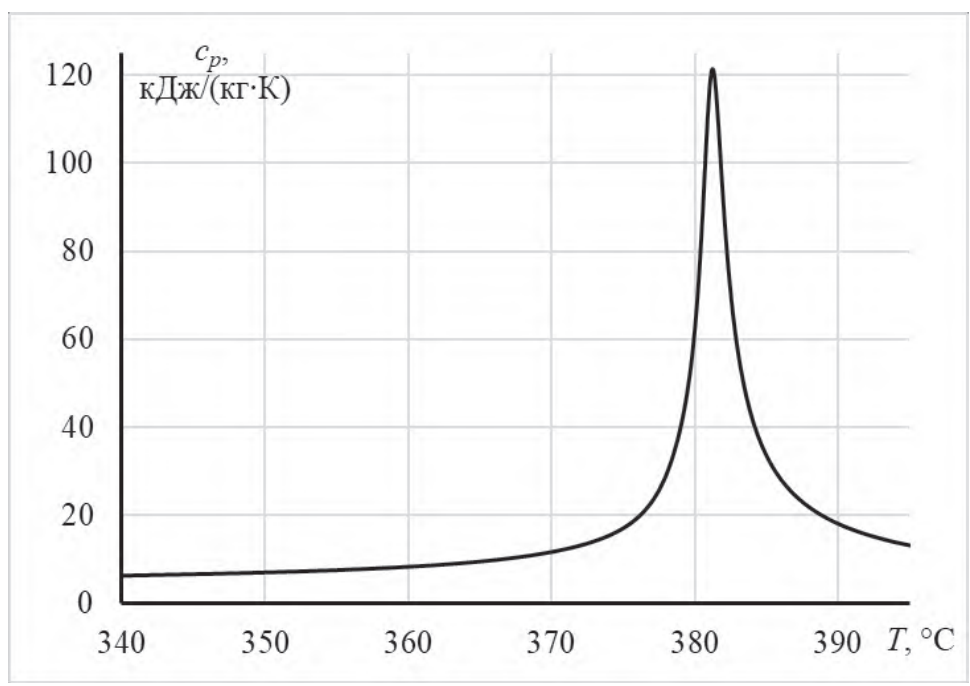

Рис. 1. Температурна залежність питомої теплоємності води при тиску $P=24,0$ МПа 
ділянці труби зі зростанням координати $x$ значення $c_{p}$ зменшуються в усіх точках перерізу $x=$ const, що відповідає переходу надкритичної води у псевдогазовий стан з більш високими температурами.

Щодо підобласті $x<x_{p c}$, то тут місце положення максимуму $c_{p}$ залежить від координати $x$. Чим менше значення х, тим ближче до стінки розташовується максимум $c_{p}$ (криві 1-5 на рис. 2a). Такий характер зміни радіального розподілу $c_{p}$, як очевидно, пов'язаний з тим, що вниз за потоком до температури псевдокритичного переходу нагріваються все більш віддаленні від стінки шари води.
Координати $r_{\max }$ розташування вказаного максимуму ср визначається перетином перерізу $x=$ const 3 ізотермою псевдокритичного переходу $T_{p c}$. На рис. 4 наведено конфігурацію цих ізотерм для різних значень $q$.

Згідно з одержаними даними, при відносно малих величинах $q(q=239$ кВт/м²) на значній ділянці труби, розташованій зліва відізотерми $T_{p c}$, температуратеплоносія нижче Трс і відповідно він перебуває у псевдорідкому стані. Зі зростанням $q(q=310$ кВт/м²) розміри цієї ділянки зменшуються. І відповідно збільшуються розміри ділянки зправа від ізотерми $T_{p c}$, де стан теплоносія визначається як псевдогазовий. Щодо координати $\mathrm{x}_{\mathrm{pc}}$, яка відповідає

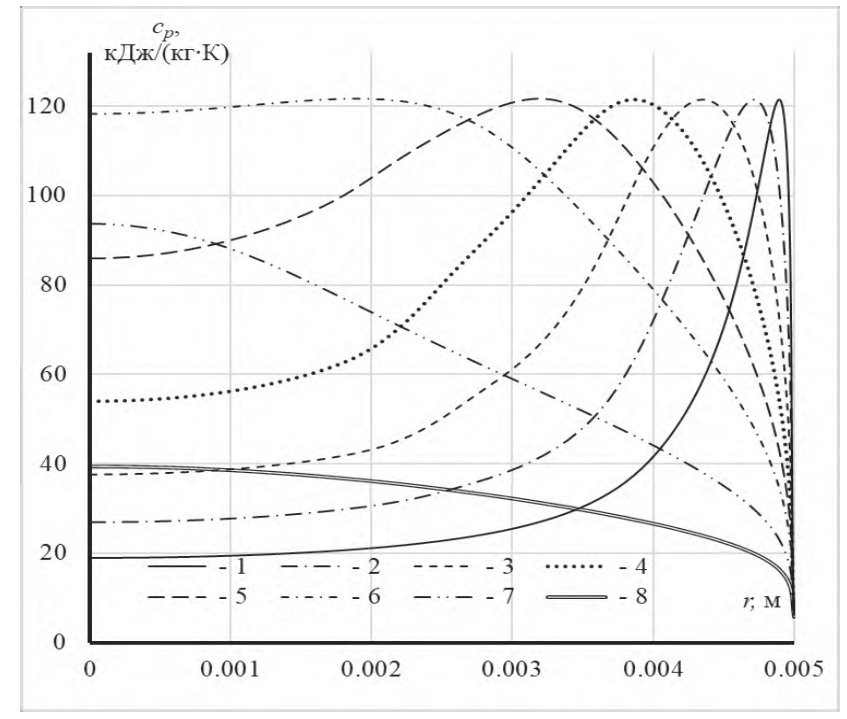

a)

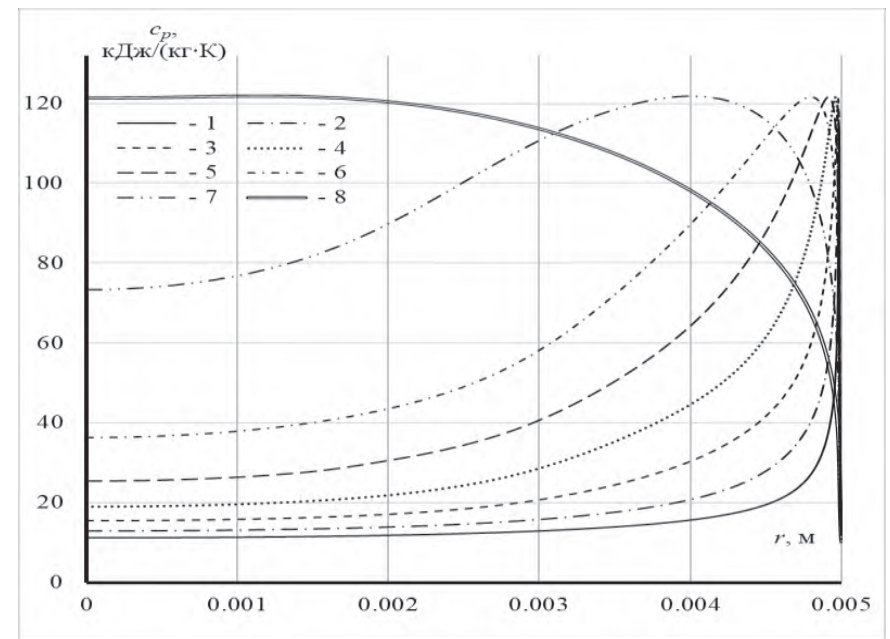

6)

Рис. 2. Розподіл питомої теплоємності надкритичної води с по радіусу труби при значеннях підведеного теплового потоку $q=310 \mathrm{\kappa Bm} / \mathrm{s}^{2}$ (a) i $q=239 \kappa \mathrm{Bm} / \mathrm{м}^{2}$ (б) на різній відстані

х від входу у нагрівану ділянку труби: $1-x=2.0 \mathrm{~m} ; 2-x=2.2 \mathrm{~m} ; 3-x=2.4 \mathrm{~m} ; 4-x=2.6 \mathrm{~m}$;

$5-x=2.8 \mathrm{~m} ; 6-x=3.0 \mathrm{~m} ; 7-x=3.4 \mathrm{~m} ; 8-x=3.8 \mathrm{~m}$. 


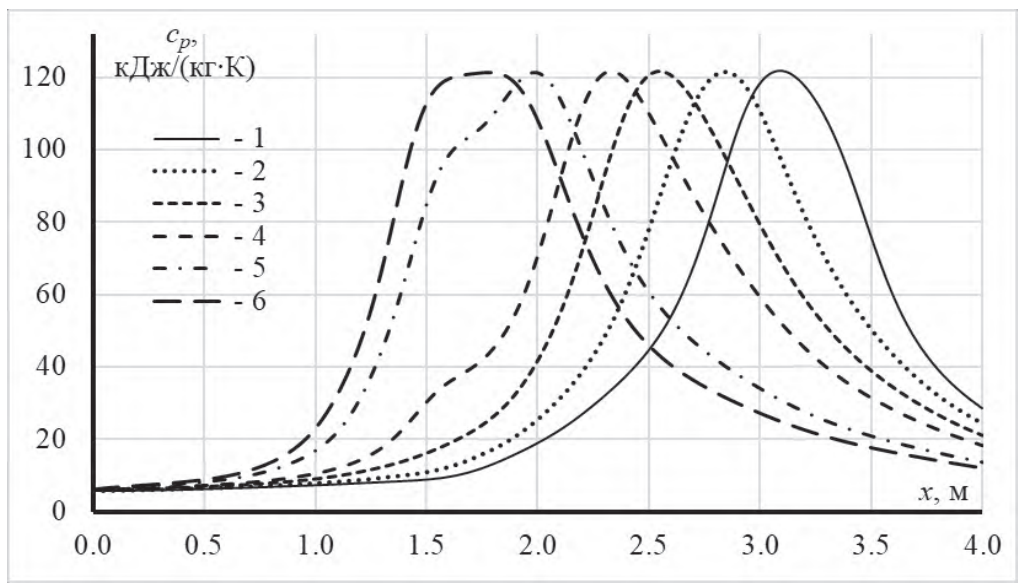

a)

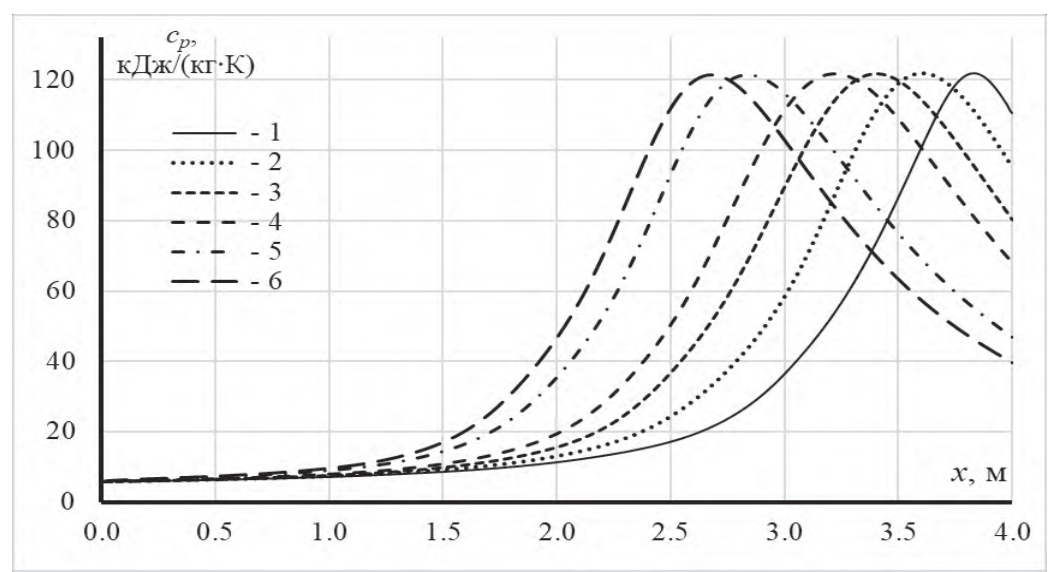

б)

Рис. 3. Розподіл питомої теплосмності надкритичної води $c_{p}$ по довжині труби при величинах підведеного теплового потоку $q=310 \mathrm{\kappa Bm} / \mathrm{M}^{2}$ (a) i $q=239 \mathrm{\kappa Bm} / \mathrm{M}^{2}$ (б) для різних значень

радіальної координати: $1-r=0.0 \mathrm{~m} ; 2-r=0.003 \mathrm{~m} ; 3-r=0.004 \mathrm{~m} ; 4-r=0.0045 \mathrm{~m}$;

$$
5-r=0.0049 \mathrm{M} ; 6-r=0.00495 \mathrm{M}
$$

положенню ізотерми $T_{p c}$ на осі труби, то вона теж суттєво залежить від величини $q$. Як видно з рис. 4 , для $q=310$ кВТ/м² значення хрс становить $3,10 \mathrm{м}$, а для $q=$ 239 кВт/м² - 3,73 м.

Відповідно до описаної конфігурації ізотерм $T_{p c}$ при різних значеннях $q$ характер зміни $c_{p}$ по радіусу труби для $q=239 \mathrm{\kappa B} / \mathrm{M}^{2}$ суттєво відрізняється від ситуації для $q=310 \kappa \mathrm{КT} / \mathrm{M}^{2}$ (рис. 2а та 2б). А саме, при $q=239 \kappa \mathrm{\kappa T} / \mathrm{M}^{2}$ зона $x<x_{p c}$, в якій залежність $c_{p}=f(r)$ має екстремальний характер, значно збільшується. А зона, $x \geq x_{p c}$, в якій відбувається падіння $c_{p}$ зі зростанням х, скорочується.

Значно відрізняються також картини розподілу $c_{p}$ по довжині труби при різних величинах теплового потоку $q$ (рис. 3а, 3б). Хоча вказані картини $є$ схожими в якісному відношенні, менше з тим вони мають суттєві кількісні відміності. При фіксованих значеннях радіуса $\mathrm{r}$ зі зменшенням $q$ координати хmax, що відповідають максимумам кривих $c_{p}=f(x)$, зміщуються в область більших значень х. (Ці координати відповідають перетину поверхні $r=$ const 3 ізотермою $T_{p c}$ ).

Насамкінець слід відзначити, що описаний складний характер просторового розподілу питомої теплоємності надкритичної води та інших фізичних властивостей зумовлює появу таких явищ, як змінність сил плавучості та прискорення потоку по довжині каналу і визначає особливості теплообміну і температурного режиму при течії за умов надкритичного тиску.

\section{Висновки.}

Iз застосуванням FLUENT коду проведено комп'ютерне моделювання течії та теплообміну 


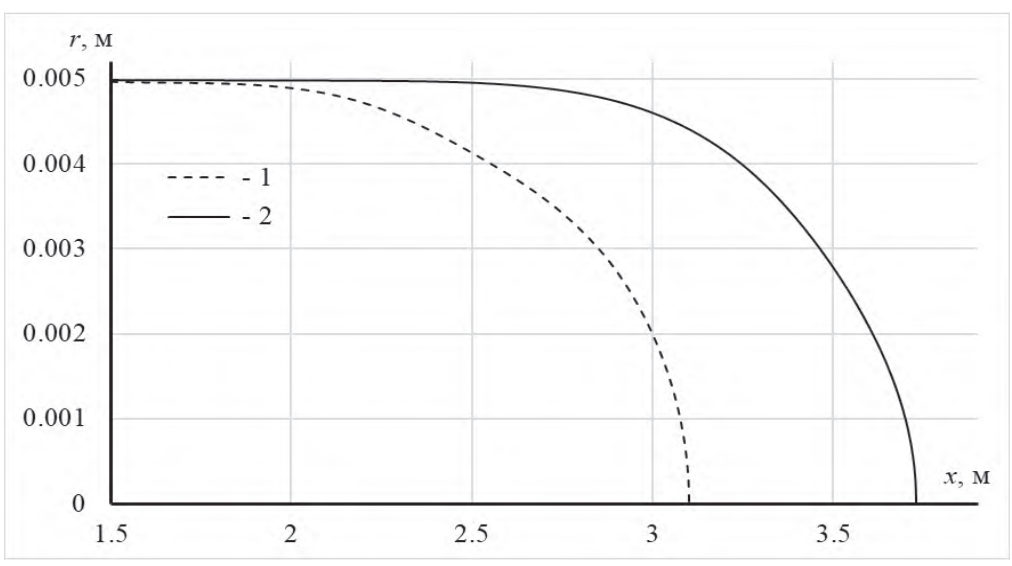

\section{Рис. 4. Конфігурація ізотерм псевдокритичного переходу $T_{p c}$ дя різних значень підведеного теплового потоку q: 1 - $310 \kappa \mathrm{Km} / \mathrm{M}^{2} ; 2-239 \kappa \mathrm{Bm} / \mathrm{M}^{2}$}

надкритичної води у вертикальних гладких трубах за умов змішаної конвекції та виконано аналіз просторового розподілу питомої теплоємності води. При цьому:

1. Проаналізовано характер конфігурації ізотерм псевдофазового переходу «псевдорідина - псевдогаз», положення яких визначає розташування максимумів питомої теплоємності води у просторовій картині іiі розподілу.

2. Встановлено, що теплоємність надкритичної води в трубі за досліджуваних умов змінюється майже у 20 разів. Досліджено особливості радіальних розподілів $c_{p}$ по довжині труби та поздовжних розподілів $c_{p}$ для різних значень радіальної координати. Визначено ефекти впливу на вказані розподіли питомої теплоємності величини теплового потоку $q$, що підводиться до стінки труби.

\section{ЛІТЕРАТУРА}

1. Фиалко Н.М., Пиоро И.Л., Майсон Н.В., Меранова Н.О. Моделирование течения и теплообмена в гладких трубах при сверхкритических давлениях. Промышленная теплотехника. 2016. 38. №3. С.10-17.

2. Zvorykin, A., Fialko, N., Meranova, N., Aleshko, S., Maison, N., Voitenko, A., and Pioro, I. Computer Simulation of Flow and Heat Transfer in Bare Tubes at Supercritical Parameters. Proceedings of the 24th International Conference On Nuclear Engineering (ICONE-24), June 2630, Charlotte, NC, USA, Paper \#60390, 2016. 12 pages.

3. Фиалко Н.М., Пиоро И.Л., Майсон Н.В., Меранова Н.О., Шараевский И.Г. Влияние массовой скорости потока на характеристики течения и теплообмена в гладких трубах при сверхкритических параметрах. Промышленная теплотехника. 2016. 38. №4. С.5-13.
4. Zvorykina A., Pieman W., Saltanov E., Grande L., Pioro I., Fialko N. Current status and future applications of supercritical pressures in power engineering. Proceedings of 20th Internetional Conference on Nuclear Engineering (ICONE-20), July 30 - August 3, 2012, Anaheim, CA, USA. $13 \mathrm{p}$.

5. Фиалко Н.М., Шеренковский Ю.В., Меранова Н.О., Алешко С.А., Стрижеус С.Н., Войтенко А.Ю., Хмиль Д.П., Брусинская Я.В., Остапчук Т.С. Характеристики теплообмена в вертикальних трубах при сверхкритических давлениях. Сборник трудов «Проблемы экологии и эксплуатации объектов энергетики» / Институт промышленной экологии. К.: ИПЦ АЛКОН НАН Украины, 2017. C.130-133.

6. Zvorykin, A., Fialko, N., Sherenkovskiy, J., Aleshko, S., Meranova, N., Hanzha, M., Bashkir, I., Stryzheus, S., Voitenko, A., and Pioro, I. (2017). CFD Study on Specifics of Flow and Heat Transfer in Vertical Bare Tubes Cooled with Water at Supercritical Pressures. Proceedings of the 25th International Conference On Nuclear Engineering (ICONE-25), July 2-6 2017, Shanghai, China, Paper \#66528, 13 pages.

7. Фіалко Н.М., Пиоро І.Л., Прокопов В.Г., Шеренковський Ю.В., Меранова Н.О., Альошко С.О. CFD моделювання теплообміну при течії води надкритичних параметрів у вертикальних гладких трубах. Промышленная теплотехника. 2018. 40. №1. С.12-20.

8. Фиалко Н.М., Прокопов В.Г., Шеренковский Ю.В., Меранова Н.О., Алешко С.А., Власенко Т.С., Шараевский И.Г., Зимин Л.Б., Стрижеус С.Н., Хмиль Д.П. Особенности изменения теплофизических свойств сверхкритической воды при течении в круглых обогреваемых трубах. Науковий вісник НЛТУ. 2018. 28. №3. С.117-121. 
9. Фіалко Н.М., НосовськийА.В., ШеренковськийЮ.В., Меранова Н.О., Шараєвський І.Г., Піоро І.Л. Особливості течії надкритичної води в умовах змішаної конвекції. Промислова теплотехніка. 2018. 40. №3. С. 12-19.

10. Fialko N., Sherenkovskii Ju., Meranova N., Aleshko S., Vlasenko T. Thermophysical properties of supercritical water at an upward flow in vertical bare channels. Міжнародна мультидисциплінарна конференція «Наука і техніка сьогодення: пріорітетні напрямки розвитку України та Польщі». м. Воломін 19-20 жовтня 2018 р. С. 116-120. ISBN 978-9934-571-55-8.

11. Фиалко Н.М., Пиоро И.Л., Шеренковский Ю.В., Майсон Н.В., Меранова Н.О., Шараевский И.Г. Влияние теплового потока на стенке канала и давления воды на характеристики течения и теплообмена в гладких трубах при сверхкритических параметрах. Промышленная теплотехника. 2016. 38. №5. С.5-13.

12. Фіалко Н.М., Носовський А.В., Шеренковський Ю.В., Меранова Н.О., Шараєвський І.Г., Піоро І.Л. CFD аналіз тепловіддачі надкритичної води в умовах змішаної конвекції. Промислова теплотехніка. 2018. 40. №4. C. 5-12.
13. Zvorykina A., Khmil D., Fialko N., Pioro I., Stryzheus S. CFD Analysis of Supercritical-Water Flow and Heat Transfer in Vertical Bare Tube 26th International Conference on Nuclear Engineering, ICONE26-81045, (October 24, 2018), V009T16A003, 14 pages.

14. Фіалко Н.М., Носовський А.В., Піоро І.Л., Шеренковський Ю.В., Меранова Н.О., Альошко С.О., Хміль Д.П., Шараєвський І.Г., Зімін Л.Б. Дослідження особливостей теплообміну надкритичної води у вертикальних гладких трубах Сборник трудов «Проблемы экологии и эксплуатации объектов энергетики». Институт промышленной экологии. К.: ИПЦ АЛКОН НАН Украины, 2019. С. 144-147.

15. National Institute of Standards and Technology, NIST Reference Fluid Thermodynamic and Transport Properties - REFPROP, NIST Standard Reference Database 23, Ver.8.0, Boulder, CO, U.S., Department of Commerce, 2007. 


\section{FEATURES OF SPATIAL DISTRIBUTION OF THE SPECIFIC HEAT CAPACITY OF SUPERCRITICAL WATER DURING ITS FLOW IN VERTICAL BARE TUBES}

\author{
Fialko N.M. ${ }^{1}$, Nosovsksyi A.V. ${ }^{2}$, Aleshko S.O. ${ }^{1}$, \\ Pioro I.L. ${ }^{3}$, Khmil D.P. ${ }^{1}$ \\ ${ }^{1}$ Institute of Engineering Thermophysics, National \\ Academy of Sciences of Ukraine, 2a, Marii Kapnist str., \\ Kyiv, 03680, Ukraine \\ ${ }^{2}$ Institute of NPP Safety Problems of NAS of Ukraine, 12, \\ Lysogirska str., Kyiv, 03028 Kyiv, Ukraine
}

${ }^{3}$ Faculty of Energy Systems and Nuclear Science University of Ontario Institute of Technology 2000 Simcoe Str. N., Oshawa ON L1K 7 K4 Canada

\section{https://doi.org/10.31472/ttpe.3.2021.2}

The results of computer modeling of the spatial distribution of the specific heat capacity under condition of the upstream flow of supercritical water in vertical bare tubes are given. The features of the motion along the tube length the front of the pseudo-phase transition "pseudoliquidpseudogas" are considered. The position of this front determines the location of the extremums of the specific heat capacity of water. The regularities of changes in the radial distributions of heat capacity along the length of the tube and longitudinal distributions for different values of the radial coordinate are investigated. The data of a comparative analysis of this distribution at various values of the specific heat flux supplied to the tube wall are presented.

References 15, figure 4.

Key words: CFD simulation, heat capacity of supercritical water, mixed convection.

1. Fialko N.M., Pioro I.L., Maison N.V., Meranova N.O. [Simulation of Flow and Heat Transfer in Bare Tubes at Supercritical Pressures]. [Industrial Heat Engineering]. 2016. 38. No. 3. P.10-17. (In Ukr.)

2. Zvorykin A., Fialko N., Meranova N., Aleshko S., Maison N., Voitenko A., and Pioro I. Computer Simulation of Flow and Heat Transfer in Bare Tubes at Supercritical Parameters. Proceedings of the 24th International Conference On Nuclear Engineering (ICONE-24), June 2630, 2016, Charlotte, NC, USA, Paper \#60390, 12 pages.

3. Fialko N.M., Pioro I.L., Maison N.V., Meranova N.O., Sharaevskiy I.G. [Influence of the Mass Flux on the Characteristics of Flow and Heat Transfer in Bare Tubes at Supercritical Parameters.], [Industrial Heat Engineering]. 2016. 38. No. 4. P.5-13. (In Ukr.)
4. Zvorykina A., Pieman W., Saltanov E., Grande L., Pioro I., Fialko N. Current status and future applications of supercritical pressures in power engineering. Proceedings of 20th Internetional Conference on Nuclear Engineering (ICONE-20), July 30 - August 3, 2012, Anaheim, CA, USA. 13 p.

5. Fialko N.M., Sherenkovskiy Ju.V., Meranova N.O., Aleshko S.A., Strizheus S.N., Voitenko A.Yu., Khmil D.P., Brusinskaya Ya.V., Ostapchuk T.S. [Characteristics of Heat Transfer in Vertical Tubes at Supercritical Pressures]. [Problems of Ecology and Operation of Energy Facilitie]. Institute of Industrial Ecology. Kyiv: IPC ALKON NAS of Ukraine. 2017. P. 130-133. (In Ukr.)

6. Zvorykin A., Fialko N., Sherenkovskiy J., Aleshko S., Meranova N., Hanzha M., Bashkir I., Stryzheus S., Voitenko A., and Pioro I. CFD Study on Specifics of Flow and Heat Transfer in Vertical Bare Tubes Cooled with Water at Supercritical Pressures. Proceedings of the 25th International Conference On Nuclear Engineering (ICONE-25), July 2-6 2017, Shanghai, China, Paper \#66528, 13 pages.

7. Fialko N.M., Pioro I.L., Prokopov V.G., Sherenkovskiy Ju.V., Meranova N.O., Aleshko S.A. [CFD Modeling of Heat Transfer for Supercritical Water Flow in Vertical Bare Tubes]. [Industrial Heat Engineering]. 2018. 40, No. 1. P.12-20. (In Ukr.)

8. Fialko N.M., Prokopov V.G., Sherenkovsky Ju. V., Meranova N.O., Aleshko S.A., Vlasenko T.S., Sharaevsky I.G., Zimin L.B., Strizheus S.N., Khmil D.P. Features of the Change in the Thermophysical Properties of Supercritical Water during Flow in Round Heated Tubes. Scientific Bulletin of UNFU. 2018. 28, №3. P.117-121.

9. Fialko N.M., Nosovskiy A.V., Sherenkovskiy Ju.V., Meranova N.O., Sharaevskiy I.G., Pioro I.L. [Features of the Flow of Supercritical Water under Conditions of Mixed Convection]. [Industrial Heat Engineering]. 2018. 40. №3. P. 12-19. (In Ukr.)

10. Fialko N., SherenkovskiiJu., MeranovaN., Aleshko S., Vlasenko T. Thermophysical properties of supercritical water at an upward flow in vertical bare channels. International multidisciplinary conference "Science and technology of the present: priority directions of development of Ukraine and Poland." M. Volomin, October 19-20, 2018. P. 116-120. ISBN 978-9934-571-55-8.

11. Fialko N.M., Pioro I.L., Sherenkovskiy Ju.V., Maison N.V., Meranova N.O., Sharaevskyi I.G. [Influence of Heat Flux on the Channel Wall and Water Pressure on the Characteristics of Flow and Heat Transfer in Bare Tubes at Supercritical Parameters]. [Industrial Heat Engineering]. 2016. 38. No. 5. P.5-13. (In Ukr.) 
12. Fialko N.M., Nosovskiy A.V., Sherenkovskiy Ju.V., Meranova N.O., Sharaevskyi I.G., Pioro I.L. CFD Analysis of Heat Transfer of Supercritical Water under Conditions of Mixed Convection. Industrial Heat Engineering. 2018. 40. №4. P. 5-12.

13. Zvorykina A., Khmil D., Fialko N., Pioro I., Stryzheus S. CFD Analysis of Supercritical-Water Flow and Heat Transfer in Vertical Bare Tube. 26th International Conference on Nuclear Engineering, (ICONE-26), October 24, 2018, V009T16A003; 14 pages.
14. Fialko N.M., Nosovskiy A.V., Pioro I.L., Sherenkovskiy Ju.V., Meranova N.O., Aleshko S.A., Khmil D.P., Sharaevskyi I.G., Zimin L.B. [Investigation of the Features of Heat Transfer of Supercritical Water in Vertical Bare Tubes]. Institute of Industrial Ecology. Kyiv: IPC ALKON NAS of Ukraine, 2019. P.144-147. (In Ukr.)

15. National Institute of Standards and Technology, NIST Reference Fluid Thermodynamic and Transport Properties - REFPROP, NIST Standard Reference Database 23, Ver.8.0, Boulder, CO, U.S., Department of Commerce, 2007. 